Article

\title{
Aqueous Dilution of Noble NPs Bulk Dispersions: Modeling Instability due to Dissolution by AF4 and Stablishing Considerations for Plasmonic Assays
}

\author{
Lorenzo Sanjuan-Navarro, Aaron Boughbina-Portolés, Yolanda Moliner-Martínez and \\ Pilar Campíns-Falcó *(D)
}

MINTOTA Research Group, Departament de Química Analítica, Facultat de Química, Universitat de Valencia, 46100 Burjassot, Spain; lorenzo.sanjuan@uv.es (L.S.-N.); abough@alumni.uv.es (A.B.-P.);

yolanda.moliner@uv.es (Y.M.-M.)

* Correspondence: pilar.campins@uv.es; Tel.: +34-96-354-3002; Fax: +34-96-354-3447

Received: 16 August 2020; Accepted: 7 September 2020; Published: 10 September 2020

\begin{abstract}
Among different nanomaterials, gold and silver nanoparticles (AuNPs and AgNPs) have become useful tools for a wide variety of applications in general, and particularly for plasmonic assays. Particle size and stability analysis are key elements for their practical applications since the NPs properties depend on these parameters. Hence, in the present work, asymmetrical flow field flow fractionation (AF4) coupled to UV-Vis and dynamic light scattering (DLS) detectors in series, has been evaluated for stability studies of citrate-capped AuNPs and AgNPs aqueous dispersions. First, experimental parameters, such as mobile phase or cross-flow rate were optimized. Sodium azide to $\mathrm{pH} 7$ for AuNPs and $\mathrm{pH} 9.2$ for AgNPs were selected as the optimum mobile phase. The analytical response of bulk dispersions of AuNPs (20,40,60 and $80 \mathrm{~nm})$ and AgNPs $(20,40$ and $60 \mathrm{~nm})$ and their dilutions have been studied. Fractograms showed a decrease on the absorbance signal in diluted dispersions as a function of time and particle size for the diluted dispersions that can be explained by dissolution in diluted dispersion since hydrodynamic diameter was constant. The results indicated that the dependence of the signal with time was more intense for AgNPs than for AuNPs, which can be correlated with its lower stability. These findings should be considered when plasmonic assays are realized. Here, assays involving non-oxidant acidic acids as use cases, were tested for several batches of NPs and considerations about their stability and operability stablished.
\end{abstract}

Keywords: AuNPs; AgNPs; asymmetrical flow field flow fractionation; stability; dispersion; dissolution; plasmonic assays

\section{Introduction}

Nanoparticles and nanotechnology have a large socio-economic impact in research and in several industrial activity areas. Specifically, gold and silver nanoparticles (AuNPs and AgNPs) are now used in many of the fields of science, analytical applications [1], medical applications [2], bioimaging [3], construction industry and sensor technologies [4]. Therefore, their study and characterization are necessary to fully understand and monitor their properties [5]. Different techniques have been used to characterize and quantify NPs in order to stablish their physical and chemical properties. Counting techniques, optical techniques and separation techniques have been proposed to supply the chemical and physical information required in different fields [6-10].

Asymmetrical flow field flow fractionation (AF4) is a continuous separation technique for particles and macromolecules that is based on hydrodynamic principles without using a stationary phase [11]. In this technique, a liquid cross-flow perpendicular to the channel drags the solute particles toward the 
accumulation wall and results in a size gradient. Diffusion back to the region of lower size acts as a counteracting force. These two counteracting forces balance each other and consequently a steady state distribution of analyte is established with the highest size at the accumulation wall that decreases toward the center of the channel [12]. Figure 1 shows the main steps involved in AF4: sample injection in the separation channel and sample focusing by a reverse flow (Figure 1a), and after fractionation (Figure 1b,c).

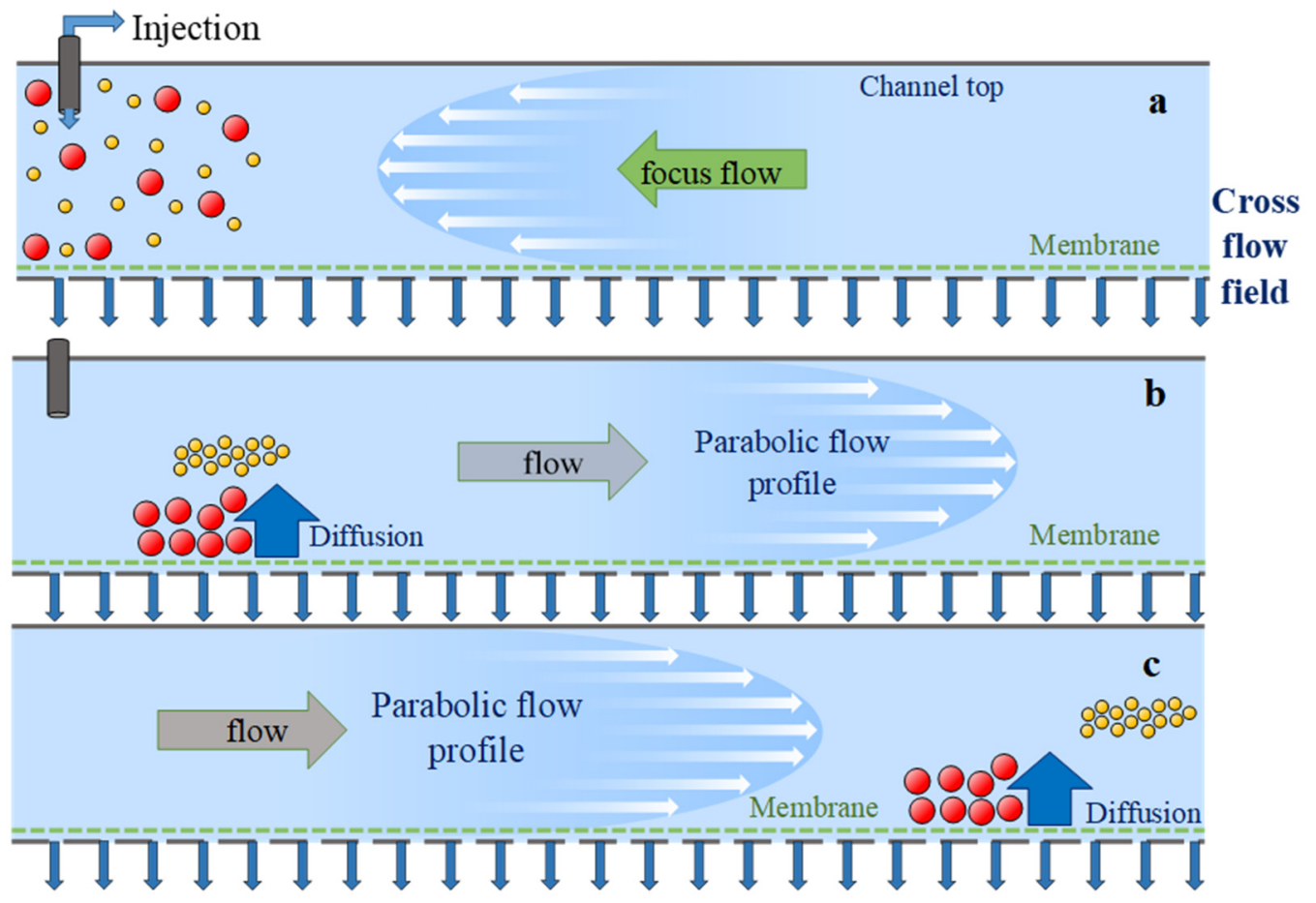

Figure 1. Schematic on the working principle of AF4: (a) Injection and sample focusing, $(\mathbf{b}, \mathbf{c})$ sample fractionation.

In particular, the metallic NPs transformations that can take place in dispersions must be considered in order to ensure the proper performance of these nanoparticles [13]. Aggregation, agglomeration, dissolution, reaction, or replacement of surface capping are potential transformations that can alter the performance and stability of metallic NPs [14,15].

Hence, in the present work, the dilution-induced variation of AuNPs and AgNPs were evaluated as a function of the dilution ratio, time, particle size, and batch. For this aim, AF4 on-line coupled with UV-Vis and DLS detector was used. Citrate-capped AuNPs and citrate-capped AgNPs with different particle size have been studied as a function of time. The main objective was to stablish the stability profiles, transformation mechanisms and therefore, the real performance of these nanomaterials for potential practical application in general and for plasmonic assays particularly. Guidelines for realizing plasmonic assays properly have been proposed. Here, plasmonic assays for hydrochloric and acetic acids are shown.

\section{Materials and Methods}

\subsection{Reagents}

AuNPs in citrate buffer solutions (average core diameter: 20 (18-22), 40 (37-43), 60 (57-63) and 80 (77-83) nm), AgNPs in citrate buffer solutions (average core diameter: 20 (16-24), 40 (36-44) and 60 (52-68) $\mathrm{nm}$ and 20 (18-22) nm) AuNPs in $0.1 \mathrm{mM}$ phosphate buffered saline (PBS) (Sigma-Aldrich, Saint Louis, MO, USA) were used, all of them $0.02 \mathrm{mg} / \mathrm{mL}$. Hydrochloric acid $(37 \% w / w)$ and concentrated acetic acid were supplied by Sigma-Aldrich and VWR Chemicals (Radnor, PA, USA), respectively. 
The liquid carrier for AF4 was prepared with sodium azide 0.02\% (NaN3, Panreac, Castellar del Vallés, Barcelona, Spain) and $\mathrm{pH}$ was adjusted using $0.1 \mathrm{M}$ sodium hydroxide ( $\mathrm{NaOH}$, Panreac). Methanol (VWR) was used for cleaning AF4 system.

3,3',5,5'-Tetramethylbenzidine (TMB) (Sigma-Aldrich) and sodium acetate anhydrous (Panreac) were also used. Moreover, it was used silver nitrate (VWR) and gold (III) chloride trihydrate (Sigma-Aldrich).

The water for all the experiments was purified through a Barnstead Nanopure II system.

\subsection{Instrumentation}

An AF2000 MT model was used to perform the AF4 measurements supplied by Postnova Analytics Inc. (Landsberg am Lech, Germany). The channel was $29 \mathrm{~cm}$ long with a $10 \mathrm{kDa}$ regenerated cellulose membrane and $350 \mu \mathrm{m}$ channel spacer. The flows were provided by two separate pumps and the cross-flow was got by a separate piston pump, which is constantly adjustable. For all AF4 analysis, the liquid carrier was high purity Mili-Q water containing $0.02 \%$ sodium azide, for AuNPs to $\mathrm{pH} 7$ and for AgNPs top H 9.2. Samples were injected using an autosampler (Postnova, Germany) and the injection volume was $20 \mu \mathrm{l}$. Optimal separation was achieved using the conditions explained in S.1 of the Supporting Information (SI) for each NPs and the flow was kept at $0.5 \mathrm{~mL} / \mathrm{min}$.

The AF4 system was coupled online with a UV-Vis detector (SPD-20AV, Postnova, Germany) and a DLS detector with temperature control (Nano-ZS, Malvern, UK). The UV-Vis detector was operated at the wavelength of $530 \mathrm{~nm}$ to AuNPs and in the interval 395-410 nm to detect AgNPs. For DLS detection, AF4 system was directly interfaced to a Zetasizer without channel split and the flow was set to $0.5 \mathrm{~mL} / \mathrm{min}$ for all fractions.

Transmission Electron Microscope (TEM) samples were prepared by delivering $10 \mu \mathrm{L}$ of the NP solution onto carbon-coated copper grid (300 mesh) and was dried overnight at room temperature. These samples were analyzed by a JEM1010 from Jeol Ltd (Akishima, Tokyo, Japan). operated at 100 kV. An Agilent (Santa Clara, CA, USA) Cary 60 UV-Vis spectrophotometer was also employed for spectroscopy studies.

\subsection{TMB Assay}

A colorimetric assay, previously reported by our group [16], was carried out to evaluate the presence of $\mathrm{Au}$ or $\mathrm{Ag}$ ions in the dispersion. $1.2 \mathrm{~mL}$ of NPs dilution dispersion was mixed with $100 \mu \mathrm{L}$ of TMB $10 \mathrm{mM}$ in EtOH and $200 \mu \mathrm{L}$ of $\mathrm{NaAc} / \mathrm{HAc}$ buffer $1 \mathrm{M}, \mathrm{pH}=4$. After $15 \mathrm{~min}$, the mixture changed a blue color complex that indicated the presence of these ions [16].

\subsection{Plasmonic Assays}

Several batches of AgNPs were diluted with ultrapure water in 1:4 proportion containing concentrations of acetic acid between 1.5 and $15 \mathrm{mM}(\mathrm{pH} \approx 3-4)$ or hydrochloric acid between 0.1 and $15 \mathrm{mM}(\mathrm{pH} \approx 2-4)$. The several dispersions were measured by UV-Vis spectroscopy and AF4.

\section{Results and Discussion}

\subsection{Characterization of Citrate-Capped-AuNPs and AgNPs Dispersions}

A study of the mass response of the AF4 was carried out for both types of NPs, the same peak areas were obtained by processing 5,10 and $20 \mu \mathrm{L}$ of diluted dispersions 1:2,1:4 and 1:8 of ultrapure water, respectively; the area values were $2.5 \pm 0.3(n=10)$ and $8.0 \pm 0.2(n=8)$ vs. for AuNPs and AgNPs, respectively. The injection volume selected for processing samples in AF4 was $20 \mu \mathrm{L}$.

Aqueous dilution of commercial citrate-capped AuNPs and AgNPs of several sizes (20, 40, 60 and $80 \mathrm{~nm}$ for AuNPs and 20, 40 and $60 \mathrm{~nm}$ for AgNPs) and at different dilution ratios were studied.

Figure 2a shows the fractograms obtained for AuNPs with different sizes at the same dilution ratio (1/4), injected just after its preparation, under the experimental conditions described in Section 2. 
It should be noted that the retention time is directly related with the nanoparticle size, larger nanoparticles experimented a greater retention into the channel as result of their minor diffusion coefficient [17]. The peak that appears at $\approx 5$ min corresponded to the void peak.
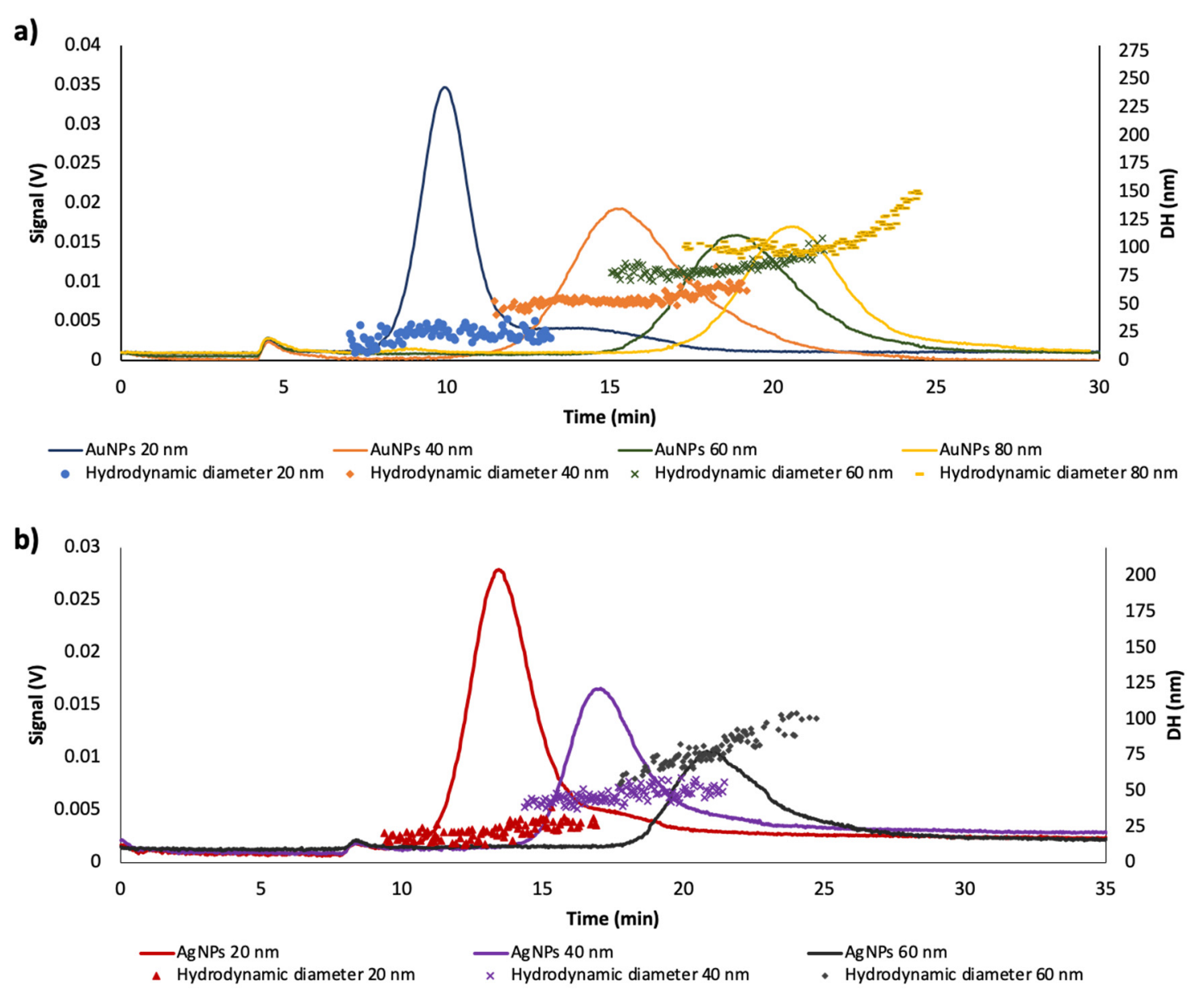

Figure 2. Fractograms for: (a) 20, 40,60 and $80 \mathrm{~nm}$ citrate-capped-AuNPs aqueous dispersions (dilution: 1/4) and (b) 20, 40 and 60 citrate-capped-AgNPs aqueous dispersions (dilution: 1/4).

The correlation between the peak area and dilution ratio (between $1 / 2$ to $1 / 10$ ) for each size were studied. Table 1 depicts the relationship between the response obtained with the UV-Vis detector and the dilution ratio. As it was expected, a lineal correlation was observed for all AuNPs sizes with satisfactory regression coefficients $\left(\mathrm{R}^{2}=0.999-0.991\right)$. Moreover, the slope, and thereof the sensitivity was higher for the smaller AuNPs at the working wavelength $(520 \mathrm{~nm})$.

Precision studies were also performed, for this aim relative standard deviation (\%RSD) was calculated in order to determine the stability of bulk dispersion for each NPs size. In this assay, four different AuNPs dispersions were prepared following the same procedure and immediately analyzed in the AF4 system. The results showed that RSD values were lower than $6.5 \%$, and therefore, intraday precision was suitable in aqueous diluted dispersions.

The same study was carried out for $\operatorname{AgNPs}(20,40,60 \mathrm{~nm})$. Figure $2 \mathrm{~b}$ shows the fractograms of these nanoparticles. The peak areas as a function of the particle size and RSD values for citrate-capped AgNPs were also calculated (Table 1). Precision was lower than that achieved by AuNPs since the RSD values were higher, near $10 \%$, although suitable for general applications, which can show the lower AgNPs' stability. 
Table 1. Values of RSD (\%) and correlation between peak area and dilution ratio for AuNPs (20, 40, 60 and $80 \mathrm{~nm})$ and AgNPs $(20,40$ and $60 \mathrm{~nm})$. A: ordinate and B: slope of the straight lines.

\begin{tabular}{|c|c|c|c|c|c|c|}
\hline & $\begin{array}{l}\text { Dilution } \\
\text { Ratio }\end{array}$ & $\begin{array}{l}\text { Concentration } \\
10^{2}(\mathrm{mg} / \mathrm{mL})\end{array}$ & $\mathbf{A} \pm \mathbf{S A}$ & $B \pm S B(\%-1)$ & $\mathbf{R}^{2}$ & RSD (\%) \\
\hline AuNPs $20 \mathrm{~nm}$ & $1 / 2$ to $1 / 10$ & 2.66 to 0.53 & $0.002 \pm 0.004$ & $0.409 \pm 0.015$ & 0.9961 & 2.2 \\
\hline AuNPs $40 \mathrm{~nm}$ & $1 / 2$ to $1 / 8$ & 2.33 to 0.58 & $0.002 \pm 0.003$ & $0.338 \pm 0.008$ & 0.9988 & 5.3 \\
\hline AuNPs $60 \mathrm{~nm}$ & $1 / 2$ to $1 / 8$ & 2.15 to 0.54 & $0.002 \pm 0.005$ & $0.262 \pm 0.014$ & 0.9942 & 6.3 \\
\hline AuNPs $80 \mathrm{~nm}$ & $1 / 2$ to $1 / 8$ & 2.03 to 0.51 & $0.000 \pm 0.005$ & $0.233 \pm 0.016$ & 0.9911 & 6.4 \\
\hline AgNPs $20 \mathrm{~nm}$ & $1 / 2$ to $1 / 8$ & 1.00 to 0.25 & $0.001 \pm 0.005$ & $0.339 \pm 0.019$ & 0.9904 & 3.1 \\
\hline AgNPs $40 \mathrm{~nm}$ & $1 / 2$ to $1 / 6$ & 1.00 to 0.33 & $0.001 \pm 0.004$ & $0.171 \pm 0.013$ & 0.9883 & 9.9 \\
\hline AgNPs $60 \mathrm{~nm}$ & $1 / 2$ to $1 / 6$ & 1.00 to 0.33 & $0.002 \pm 0.003$ & $0.119 \pm 0.009$ & 0.9890 & 10.1 \\
\hline
\end{tabular}

AuNPs' and AgNPs' recoveries from the AF4 channel were also calculated. Values between 10.3 and $29.4 \%$ for AuNPs, and between 31.6 and $65.8 \%$ for AgNPs were found. These results are in agreement with previous reports, which demonstrated that the interactions between the membrane and NPs have a high probability of undergoing irreversible interactions [18,19].

\subsection{Effect of Time in the Analytical Responses of Diluted Dispersions of NPs}

The environment of metallic NPs is a key parameter for their practical application, since their performance will depend on their stability. In several application, dilution of these NPs are needed; however, dilution-induced changes, mainly on the surface interactions that may affect the performance of this NPs. These changes depend on the kinetic of individual NPs subject to local variations and thereof, the time is an important parameter. To prove this, citrate-capped AuNPs diluted dispersions $(1 / 4)$ of different sizes were measured at different times after their dilution. Figure 3 shows the fractograms obtained at different times after dispersion preparations $(t=0,24,48$ and $72 \mathrm{~h})$. As can be seen in Figures 3a-d and 4a, there was a decrease in the absorbance signal with time, and that decrease, depended on the particle size.

The hydrodynamic diameter of the different AuNPs was measured as a function of time with the DLS detector coupled on-line with the UV-Vis detector. Figure $4 \mathrm{~b}$ shows the results obtained for the different sized NPs. As can be seen, the hydrodynamic diameter was constant over the dilution, in addition it was constant with the time and this effect was observed for all NPs sizes (DLS graphics can be seen in S.2 of the SI).

The same study was carried out for citrate-capped AgNPs in order to check whether the nature of NPs had some influence on the previous results. Figure 3e-g represents the signal variation for dispersions with different sizes (20, 40 and $60 \mathrm{~nm}$ ). As in the case of citrate-capped AuNPs, there was a decrease on the signal with time, and that decrease was a function of the particle size. Indeed, the analytical response for $60 \mathrm{~nm}$ AgNPs at $24 \mathrm{~h}$ was negligible.

However, compared with AuNPs, the increment of that decrease was higher for AgNPs (see Figure 4b), which is consistent with the higher stability of AuNPs than that given by AgNPs [20,21]. Figure $4 c, d$ shows the variation of the hydrodynamic diameter for both NPs being constant with time for both of them. A diluted aqueous dispersion of AuNPs in PBS (dilution 1/8) was measured as a function of time. As can be noted in the Supporting Information (S.3), it was observed the same effect that in previous assays for citrate-capped NPs.

Figure 5 shows the evolution of signal as function of dilution preparation time for mixtures of different sizes of AuNPs with dilution (1/8) and AgNPs with dilution (1/4). As it was expected, fractograms of citrate-capped AuNPs mixture $(20,40$ and $80 \mathrm{~nm})$ were resolved, and three peaks were obtained corresponding to 20, 40 and $80 \mathrm{~nm}$ AuNPs. As above mentioned, $24 \mathrm{~h}$ after the dilution preparation, the UV-Vis signal changed as a function of the particle size, in accordance with the results obtained for individual NPs; NPs with higher diameter experienced a decrease on the peak area higher than that provided by smaller NPs. This effect was more drastic after $48 \mathrm{~h}$ as it can be seen in Figure $5 \mathrm{a}$. 

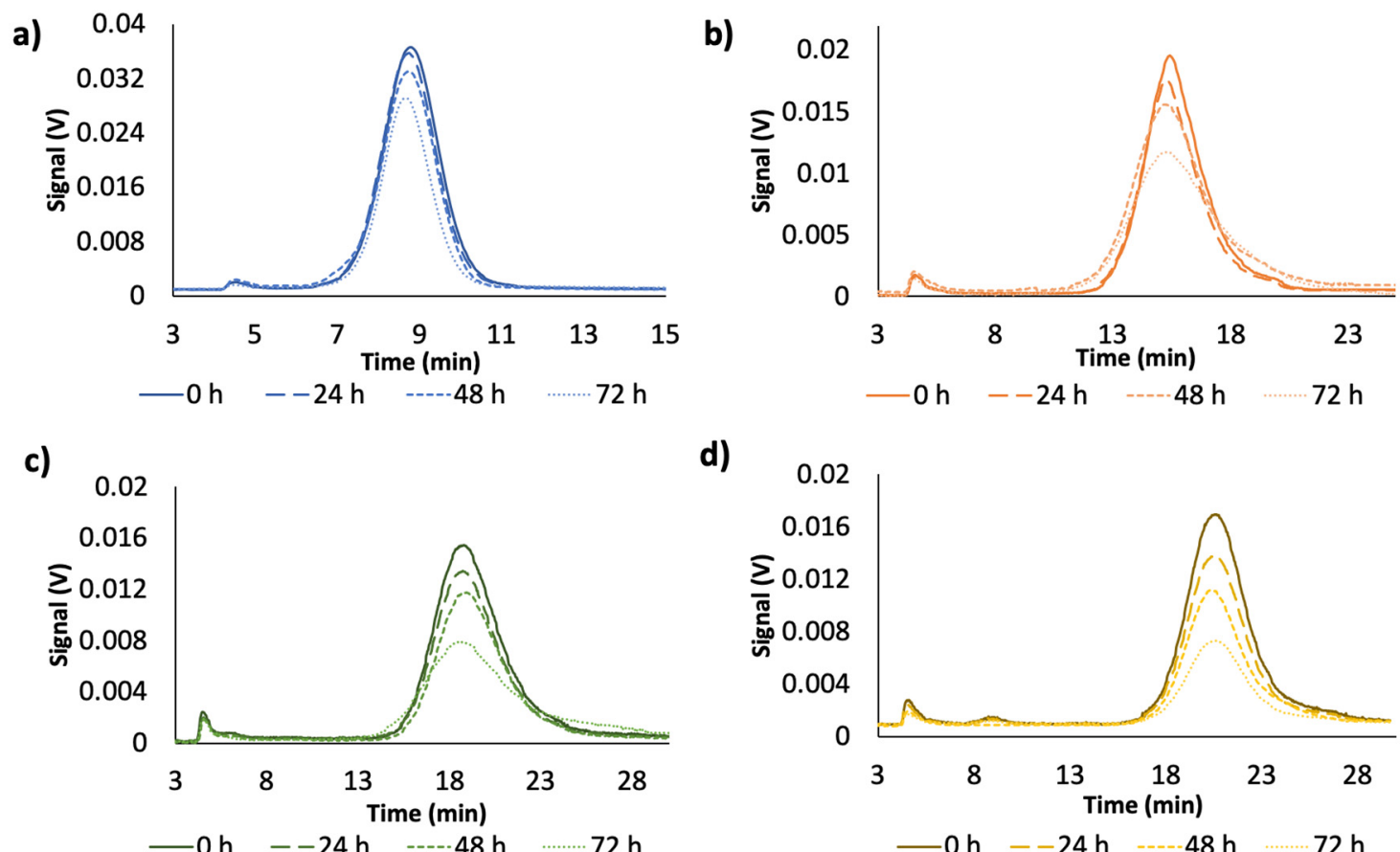

d)
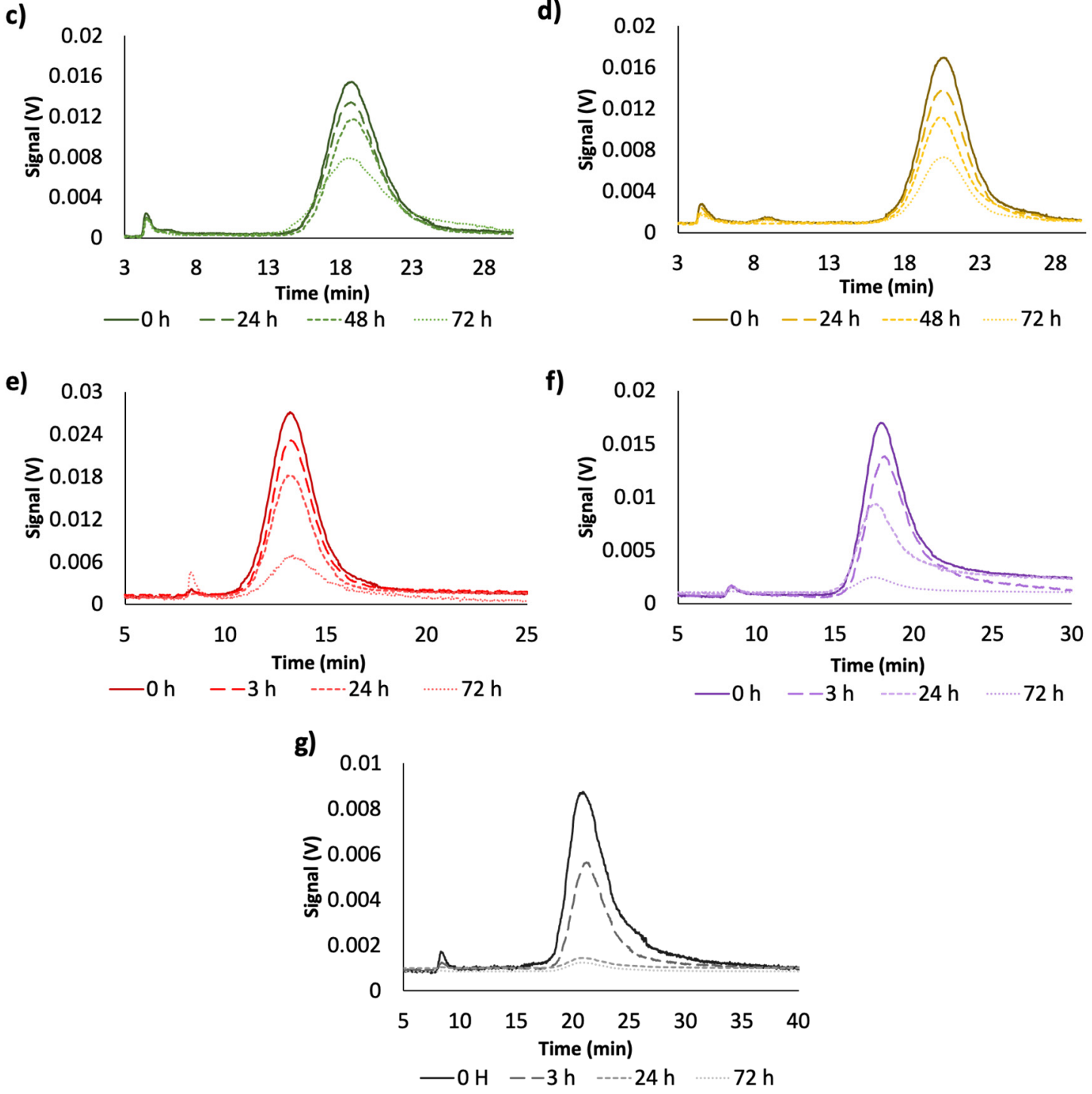

Figure 3. Fractograms of the evolution of nanoparticles dispersions as function of dilution preparation time for different sizes (a) AuNPs 20 nm, (b) AuNPs 40 nm, (c) AuNPs 60 nm, (d) AuNPs 80 nm, (e) AgNPs $20 \mathrm{~nm}$, (f) AgNPs $40 \mathrm{~nm}$ and (g) AgNPs $60 \mathrm{~nm}$. 
a)

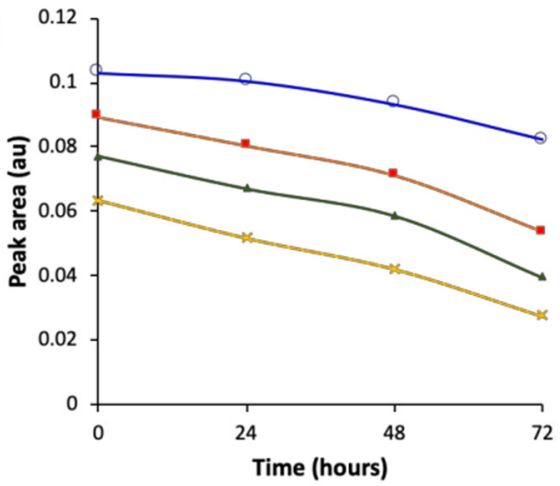

$-20 \mathrm{~nm}$ - AuNPs $\rightarrow-40 \mathrm{~nm}$-AuNPs $\longrightarrow 60 \mathrm{~nm}$-AuNPs $\rightarrow-80 \mathrm{~nm}$-AuNPs

b)

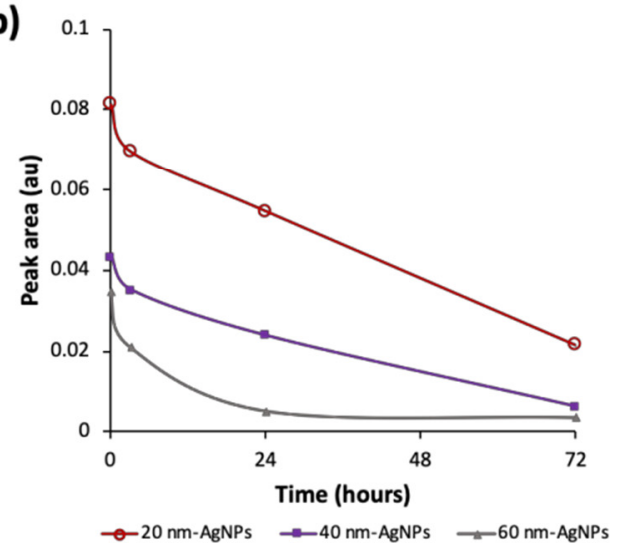

c)

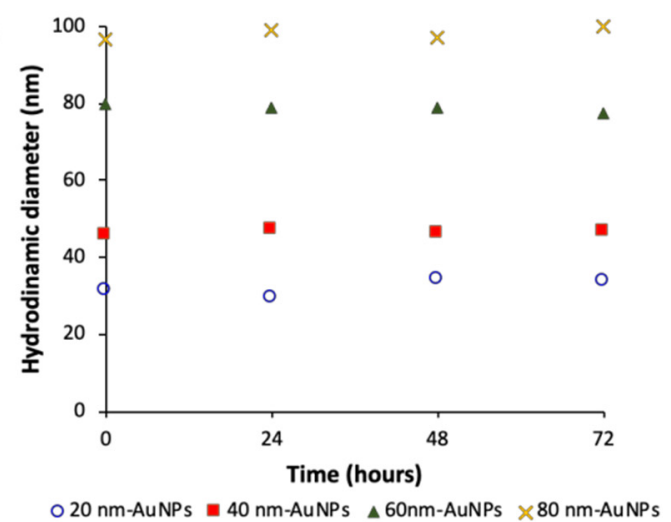

d)

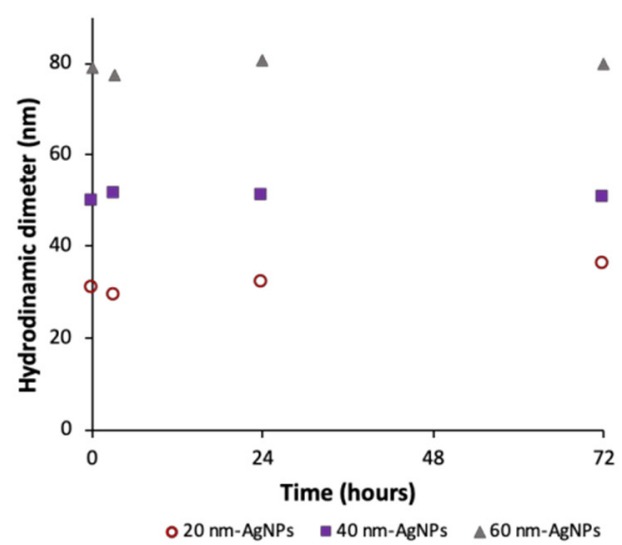

Figure 4. (a) Variation of the peak area as a function of time for citrate-capped-AuNPs. (b) Variation of the hydrodynamic diameter as a function of time for citrate-capped-AuNPs. (c) Variation of the peak area as a function of time for citrate-capped-AgNPs and, (d) Variation of the hydrodynamic diameter as a function of time for citrate-capped AgNPs.

In the case of the AgNPs mixture (20,40, and $60 \mathrm{~nm}$ ) the same evolution that that described for AuNPs was observed (Figure 5c). However, the lower stability of AgNPs due to interactions with medium, produced a more pronounced signal reduction, since dissolution in this case was more favored than for AuNPs. Indeed, the peak for $60 \mathrm{~nm}$ citrate-AgNPs was not detected after $24 \mathrm{~h}$. The average hydrodynamic diameters of dispersed NPs were stable during the whole analysis (see Figure $5 b, d$ ), as previously stated for individual particles. These results could support the hypothesis that dissolution is the mechanism by which the stability of dispersed NPs in diluted dispersions varied as a function of time.

Figure 6 summarizes the changes for $60 \mathrm{~nm}$ AuNPs as an example. As can be seen, there is a decrease on the signal, and after $72 \mathrm{~h}$, the dilution ratio varied from $1 / 4$ to $1 / 7$, in the case of this NPs (Figure 6a), but its size was constant (Figure 6c). TEM analysis showed that the NPs core size did not vary (Figure 6b). Similar considerations can be done for the other NPs analyzed. 


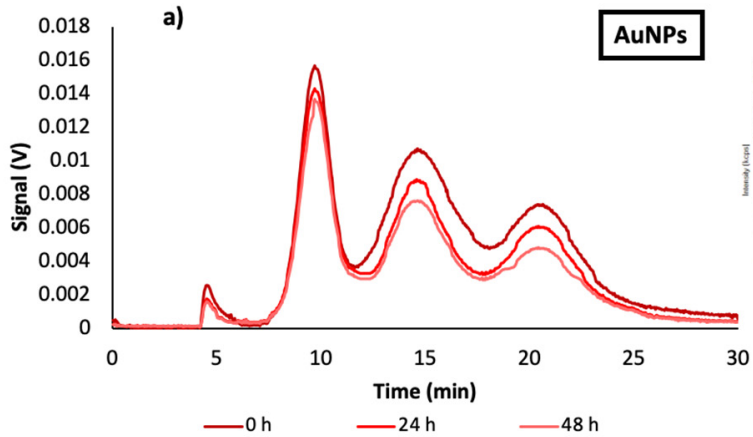

b)
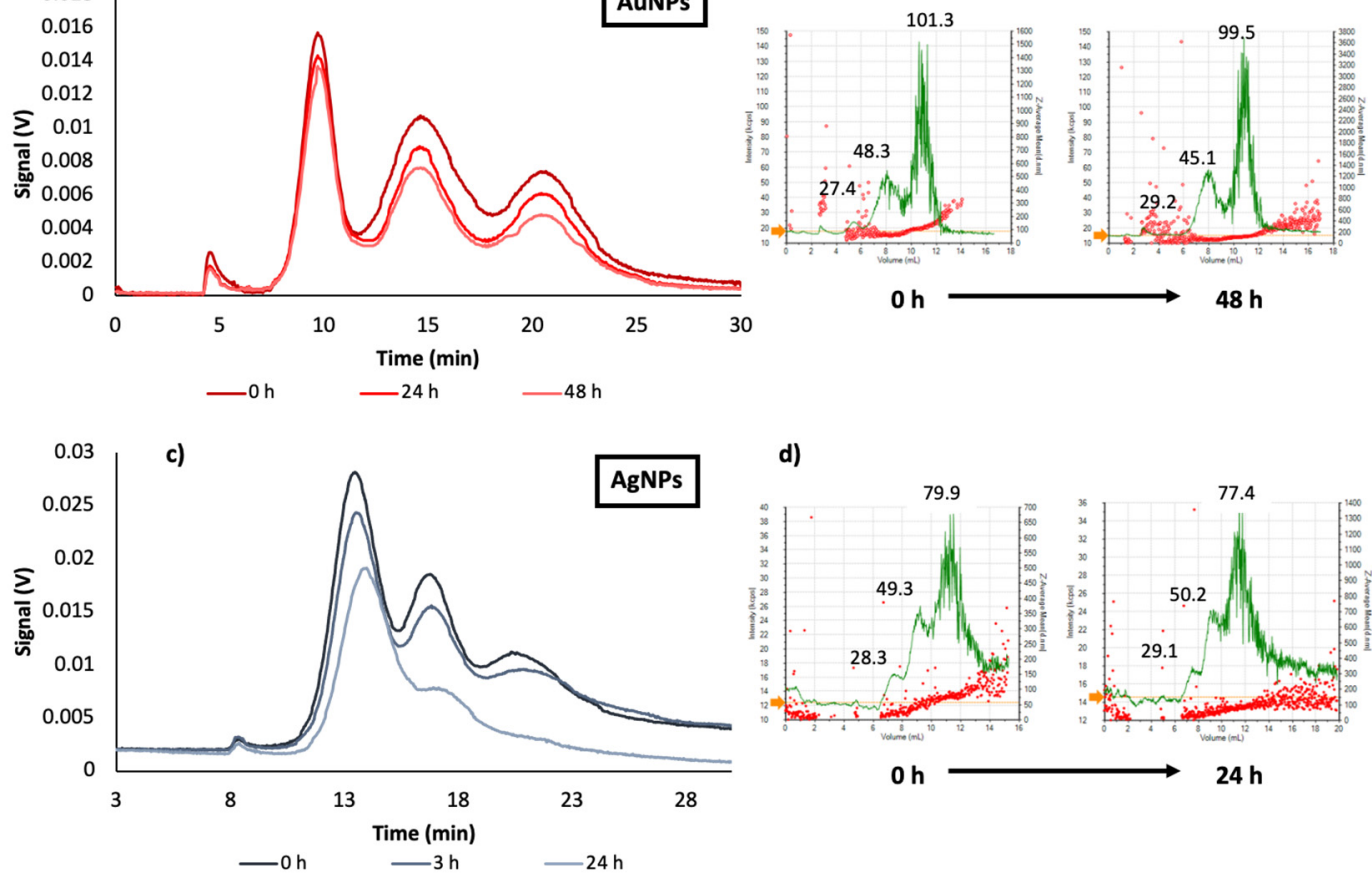

d)

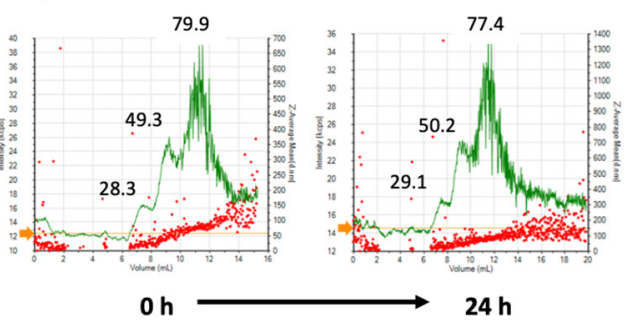

Figure 5. Mixtures of different sizes of AuNPs (dilution 1/8) and AgNPs (dilution 1/4). (a) Fractograms of the evolution of 20, 40 and $80 \mathrm{~nm}$ AuNPs mixture; (b) DLS values of AuNPs sizes, DLS fractograms in green and hydrodynamic diameters distribution in red; (c) fractograms of the evolution of 20, 40 and $60 \mathrm{~nm}$ AgNPs evolution mixture; (d) DLS values of AgNPs sizes, DLS fractograms in green and hydrodynamic diameters distribution in red.

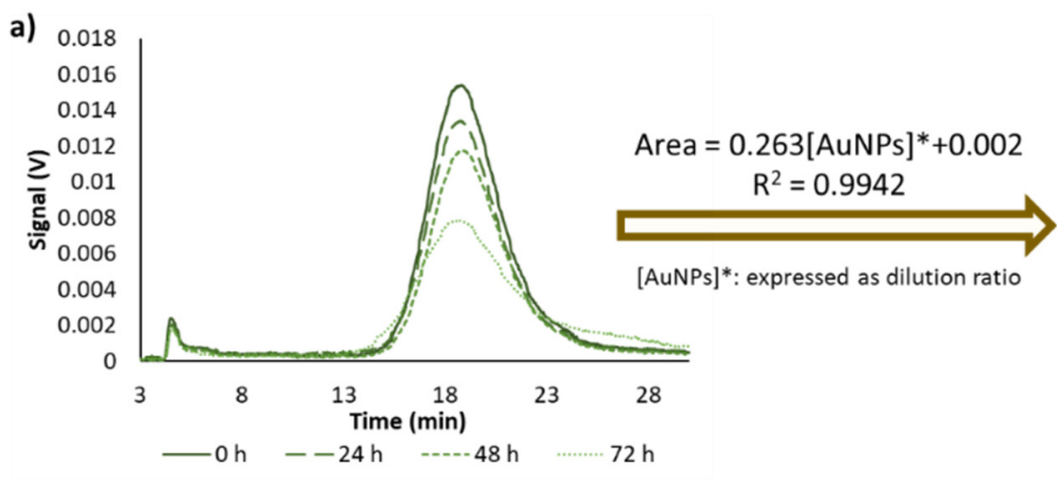

b)

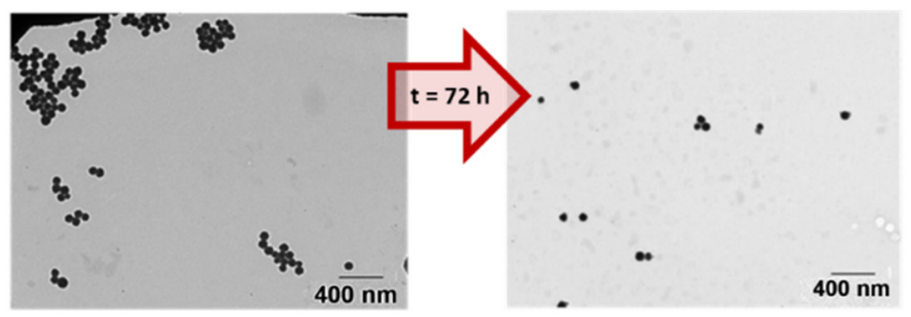

c)

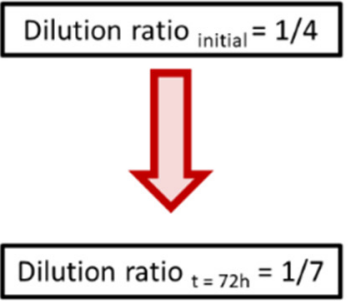

\begin{tabular}{|c|c|}
\hline Time $(\mathrm{h})$ & $\mathbf{d}_{\text {hydrodynamic }}(\mathrm{nm})$ \\
\hline 0 & 79.7 \\
\hline 24 & 80.2 \\
48 & 80.9 \\
72 & 81.6 \\
\hline
\end{tabular}

Figure 6. (a) Effect in the dilution ratio for AuNPs $60 \mathrm{~nm}$ with time. (b) TEM micrographs for diluted dispersions of citrate-capped-AuNPs $(60 \mathrm{~nm})$ just after their preparation and after $72 \mathrm{~h}$. (c) Hydrodynamic diameter of AuNPs at different dilution times (0, 24, 48 and $72 \mathrm{~h}$ ).

A TMB assay [16] was applied in order to corroborate the instability by dissolution of the dispersions. In this assay, $\mathrm{Au}$ and Ag ions can be detected in NPs dispersions, since the cationic species 
reacts with TMB to form a blue color compound $(\lambda \max =650 \mathrm{~nm})$. The first spectra of Figure $7 \mathrm{a}, \mathrm{b}$ show those obtained for just prepared diluted dispersions of AuNPs and AgNPs before and after the reaction with $\mathrm{TMB}$, respectively. These spectra were compared with the spectra obtained for $\mathrm{Au}^{3+}$ and $\mathrm{Ag}^{+}$standards after TMB derivatization as control experiments and blank solution of the TMB assay. As can be seen, cationic species were not detected for recently diluted dispersions, since there was not band at $650 \mathrm{~nm}$, and only their plasmon bands were observed. Several diluted dispersions of NPs were prepared from the batch NPs, and processed by the TMB assay after different preparation times (see Figure 7a,b). The absorbance at $650 \mathrm{~nm}$ increased with the dispersion dilution time, which is correlated with the presence of $\mathrm{Au}^{3+}$ and $\mathrm{Ag}^{+}$, indicative of dissolution of NPs.
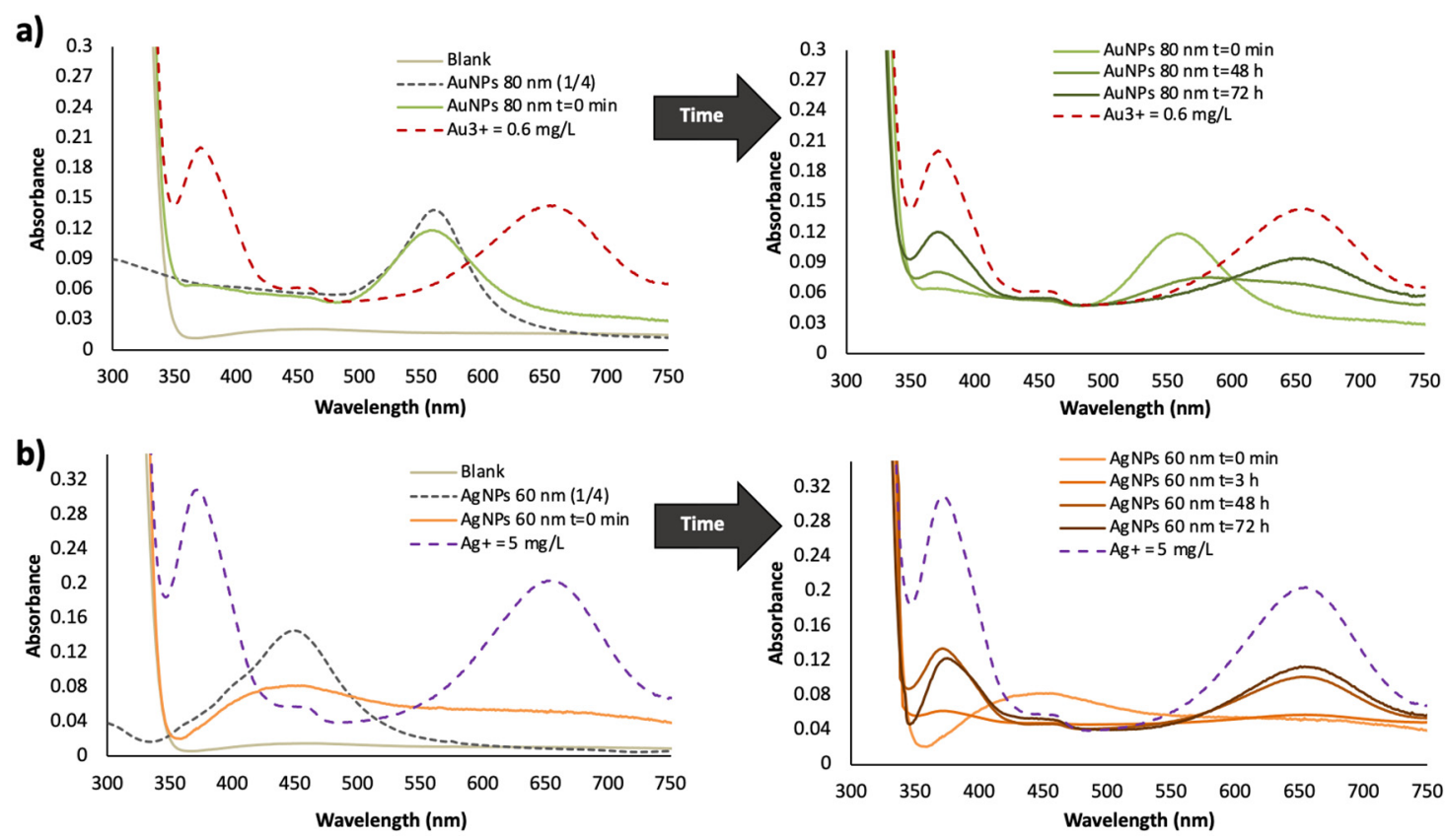

Figure 7. Gold and silver nanoparticles dissolution TMB assay for several times of preparation of diluted dispersions from batch NPs: (a) AuNPs for 0, 48 and $72 \mathrm{~h}$ and (b) 0, 3, 48 and $72 \mathrm{~h}$. For more explanation see text.

Figure $7 \mathrm{~b}$ shows the spectra for AgNPs, which indicates that the dissolution of this type of NPs is bigger than that achieved by AuNPs (see Figure 7a). This assay supported also dissolution as the reason of the loss of NPs with time for diluted dispersions.

From these results, changes of AuNPs and AgNPs with time must be taken into account since the dispersed fraction of NPs available in diluted dispersions varies with time, and therefore practical application of NPs in general must consider this effect in order to obtain reliable results. Besides, the main analytical parameters in particular, can be wrong if diluted dispersion are not deeply understood. If the fraction of NPs in diluted dispersions changes, for example limit of detection (LOD) will vary as a function of time, and this must be taken into account in order to characterize an analytical method. To demonstrate this, Table 2 shows the variation in the detection limits calculated for the diluted dispersions used after $72 \mathrm{~h}$ of its preparation, and these values are compared with the LOD calculated for diluted dispersions just prepared from the batch material. LOD was calculated by establishing the dilution ratio of a dispersion providing a signal corresponding to three times the instrumental noise from the equation signal vs. dilution ratio for each size, and taking into account the initial concentration. 
Table 2. Detection limits calculated for each diluted dispersions of citrate-capped AuNPs and AgNPs.

\begin{tabular}{ccccc}
\hline & & Co $(\mathbf{m g} / \mathbf{m L})$ & LOD $(\mu \mathrm{g} / \mathbf{m L}) \mathbf{t}=\mathbf{0} \mathbf{~ m i n}$ & LOD $(\mu \mathrm{g} / \mathbf{m L}) \mathbf{t}=\mathbf{7 2}$ \\
\hline \multirow{4}{*}{ AuNPs } & $20 \mathrm{~nm}$ & 0.053 & 0.22 & 0.26 \\
& $40 \mathrm{~nm}$ & 0.047 & 0.12 & 0.17 \\
& $60 \mathrm{~nm}$ & 0.043 & 0.14 & 0.21 \\
& $80 \mathrm{~nm}$ & 0.041 & 0.06 & 0.09 \\
\hline \multirow{3}{*}{ AgNPs } & $20 \mathrm{~nm}$ & 0.020 & 0.10 & 0.18 \\
& $40 \mathrm{~nm}$ & 0.020 & 0.23 & 0.43 \\
& $60 \mathrm{~nm}$ & 0.020 & 0.47 & 0.89 \\
\hline
\end{tabular}

\subsection{Plasmonic Assays}

From the previous results concerning stability of the citrate-capped gold and silver NPs, we used freshly diluted dispersions for plasmonic assays. Three different batches of AgNPs $20 \mathrm{~nm}$ selected as a use case were assayed. Figure 8 shows the fractograms and their spectra, as it can be seen two of them (batches 1,2) were similar, but the responses of the other (batch 3) were so different by AF4 with both UV-Vis and DLS detectors. A displacement of the retention time of the maximum of the peak is seen up to $16 \mathrm{~min}$ for batch 3, compatible with the presence of larger NPs, as well as a considerable decrease in their signal at $\lambda=395 \mathrm{~nm}$, which can respond to a lower mass of AgNPs. In addition, the DLS data determine a mean hydrodynamic diameter around $2 \mathrm{~nm}$ greater than those of the rest of the batches, as shown in Table 3. By UV-Vis spectroscopy, all batches present a similar surface plasmon band (SPB), although batch 3 shows certain differences, since it provides somewhat lower absorption and a slight bathochromic shift, which could be consistent with slightly larger AgNPs.
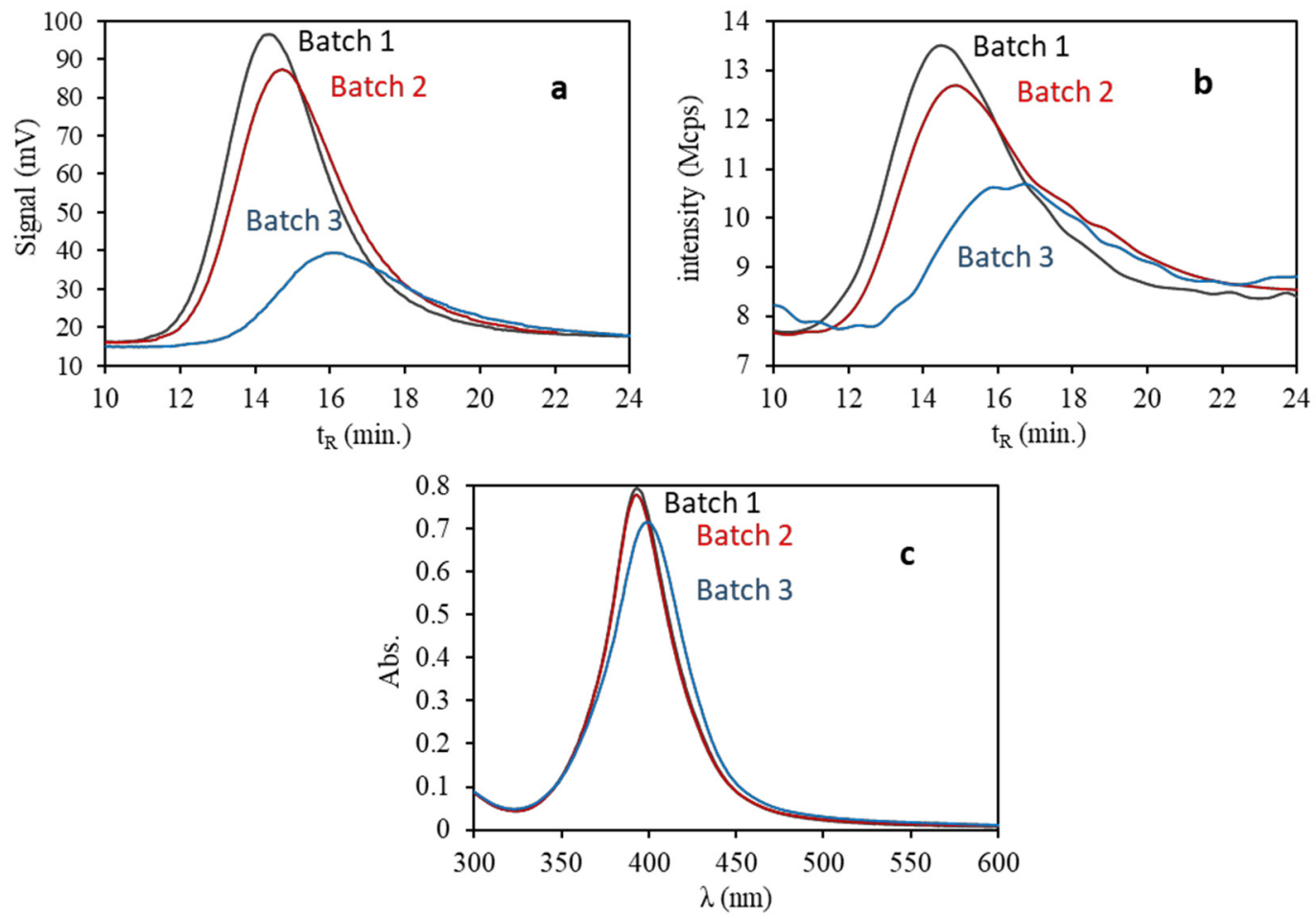

Figure 8. Fractograms of three different batches of AgNPs $20 \mathrm{~nm}$ diluted 1:4 with ultrapure water $(5 \mu \mathrm{g} / \mathrm{mL}$ ) with UV-vis detection (a) and DLS detection (b) and their spectra (c). 
Table 3. Wavelength values $(\lambda)$ of the plasmon maximum, width of the peak at half the maximum, extinction coefficient ( $\varepsilon$ max) obtained by UV-Vis spectroscopy and hydrodynamic diameter (DH) by DLS for three different commercial batches of AgNPs.

\begin{tabular}{|c|c|c|c|c|}
\hline Batch & $\lambda(\mathrm{nm})$ & Width $_{1 / 2}(\mathrm{~nm})$ & $\varepsilon \max \left(\mathrm{mM}^{-1} \cdot \mathrm{cm}^{-1}\right)$ & $\mathrm{DH}^{\mathrm{a}}(\mathrm{nm})$ \\
\hline 3 & $397.6 \pm 2.4$ & $50.2 \pm 0.1$ & $15.43 \pm 0.02$ & $22.3 \pm 2.4$ \\
\hline 2 & $393.4 \pm 2.0$ & $42.7 \pm 0.2$ & $16.83 \pm 0.05$ & $20.5 \pm 3.1$ \\
\hline 1 & $393.1 \pm 2.2$ & $42.8 \pm 0.2$ & $17.05 \pm 0.03$ & $20.1 \pm 3.0$ \\
\hline
\end{tabular}

The fractogram recorded using the DLS detector signal indicates a decrease in the intensity of the scattered light for batch 3 with respect to the rest (see Figure 8b), which signifies the presence of a smaller quantity of AgNPs. Phenomena such as dissolution and/or passivation could be considered to be a consequence of prolonged exposure to atmospheric conditions of batch 3 [22-25]. A part of the AgNPs could be dissolved and released in the form of $\mathrm{Ag}^{+}$, or it could be oxidized, generating a surface film of Ag2O, whose formation can be compatible by a bathochromic shift, as well as a lower height and broadening of the plasmon by UV-Vis spectroscopy (see Table 3 and Figure 8c) [22,24,25]. In this case, the main differences between batches could be related with the time that each of them has been exposed to the atmosphere, and consequently, with the renewal of dissolved oxygen in the suspension. If the AF4 records are compared with those obtained by UV-Vis spectroscopy, it should be noted that the AF4 technique shows a greater discrimination capacity in the characterization of aqueous dispersions of AgNPs, providing besides more information about mass and size.

We selected batch 1 for plasmonic assays involving hydrochloric and acetic acids. Figure 9 shows the spectra obtained by UV-Vis spectrometry corresponding to these assays at $10 \mathrm{~min}$, which are compatible with aggregation processes. By decreasing the $\mathrm{pH}$ of the medium, a reduction in the height of the plasmon can be observed accompanied by an increase in the absorption at higher wavelengths until the formation of a second peak whose wavelength undergoes a bathochromic shift with time and with the concentration of acid used. For hydrochloric assay, the disappearance of this second peak is observed to give rise to a continuous band of absorption related with the formation of large-sized NPs that can be part of a polydisperse suspension of AgNPs of a wide range of sizes capable of scattering light from the visible spectrum and near-infrared range (NIR). The footprints of two acids were different, the changes for acetic acid are slower that those shown for hydrochloric acid by assaying the same concentrations (between 1.5 and $15 \mathrm{mM}$ ), which can be related by its acid-base strength.

On the other hand, we studied the kinetics of the aggregation by AF4, Figure 10 shows the fractograms corresponding to the dispersion of AgNPs in the presence of 1.5 and $3.0 \mathrm{mM}$ of acetic acid at several times since its preparation. The time evolution of the suspension containing $3.0 \mathrm{mM}$ is more severe than that presented by the suspension containing $1.5 \mathrm{mM}$. By reducing the $\mathrm{pH}$, a decrease in the height of the peak with time is observed in the fractograms recorded using the UV-Vis detector (Figure 10a,c), as well as a slight increase in retention times. The fractograms recorded using the DLS detector (Figure 10b,d) show a significant increase in retention time with time, as well as a slight increase in peak intensity, which indicate a shift in the size distribution of the AgNPs toward greater dimensions. These observations are compatible with aggregation of AgNPs, since there is a decrease in the intensity of the plasmon wavelength and a growth with time in the size of the NPs, as it can be seen in the hydrodynamic diameter data in Figure 10b,d. Likewise, a higher speed is observed, as well as a larger size of the aggregates when the $\mathrm{pH}$ decreases [26-30]. 

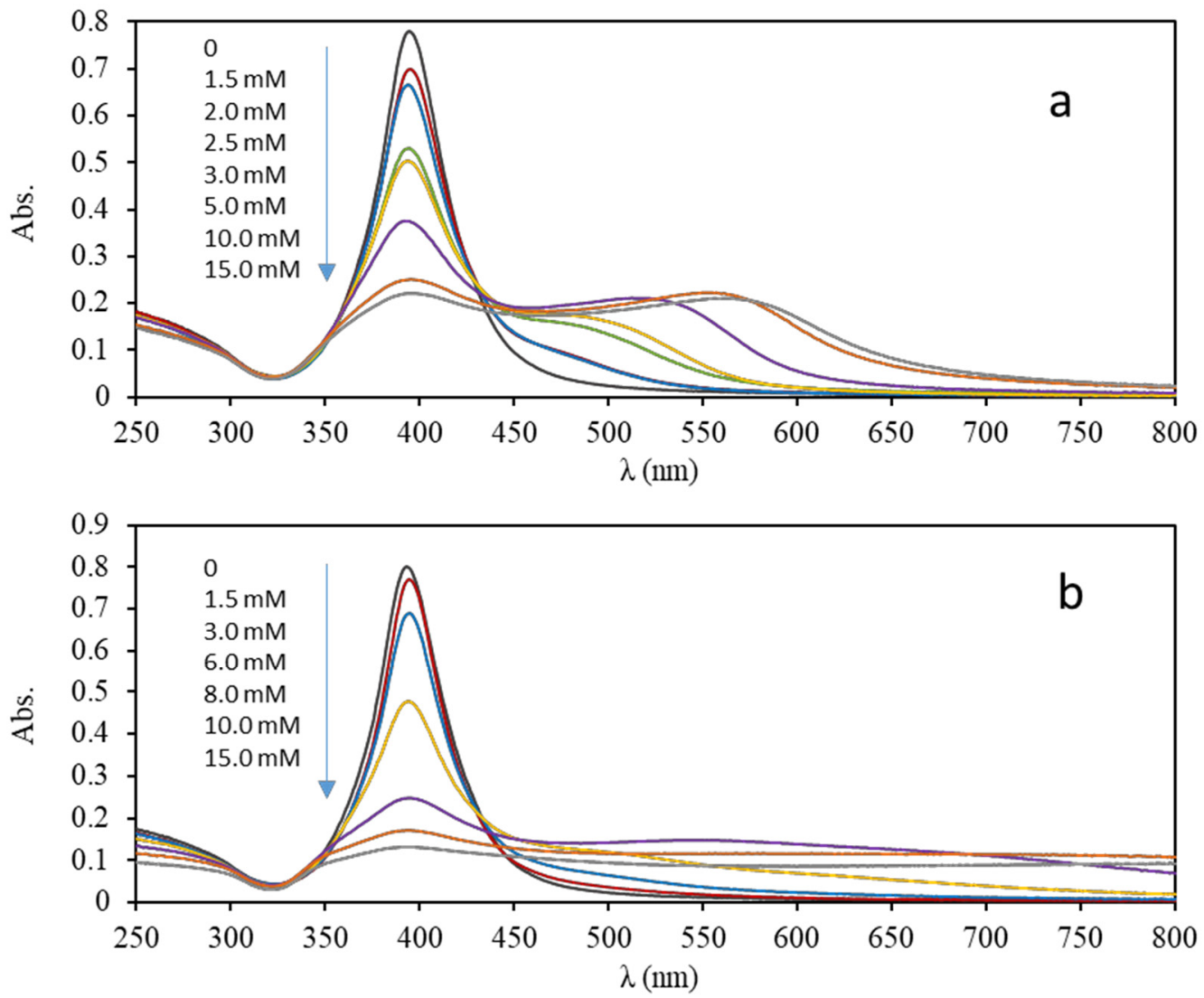

Figure 9. UV-Vis spectra recorded after $10 \mathrm{~min}$. corresponding to the suspension of AgNPs in a 1: 4 dilution $(5 \mu \mathrm{g} / \mathrm{mL})$ in the presence of different concentrations of acetic acid (a) and hydrochloric acid (b).
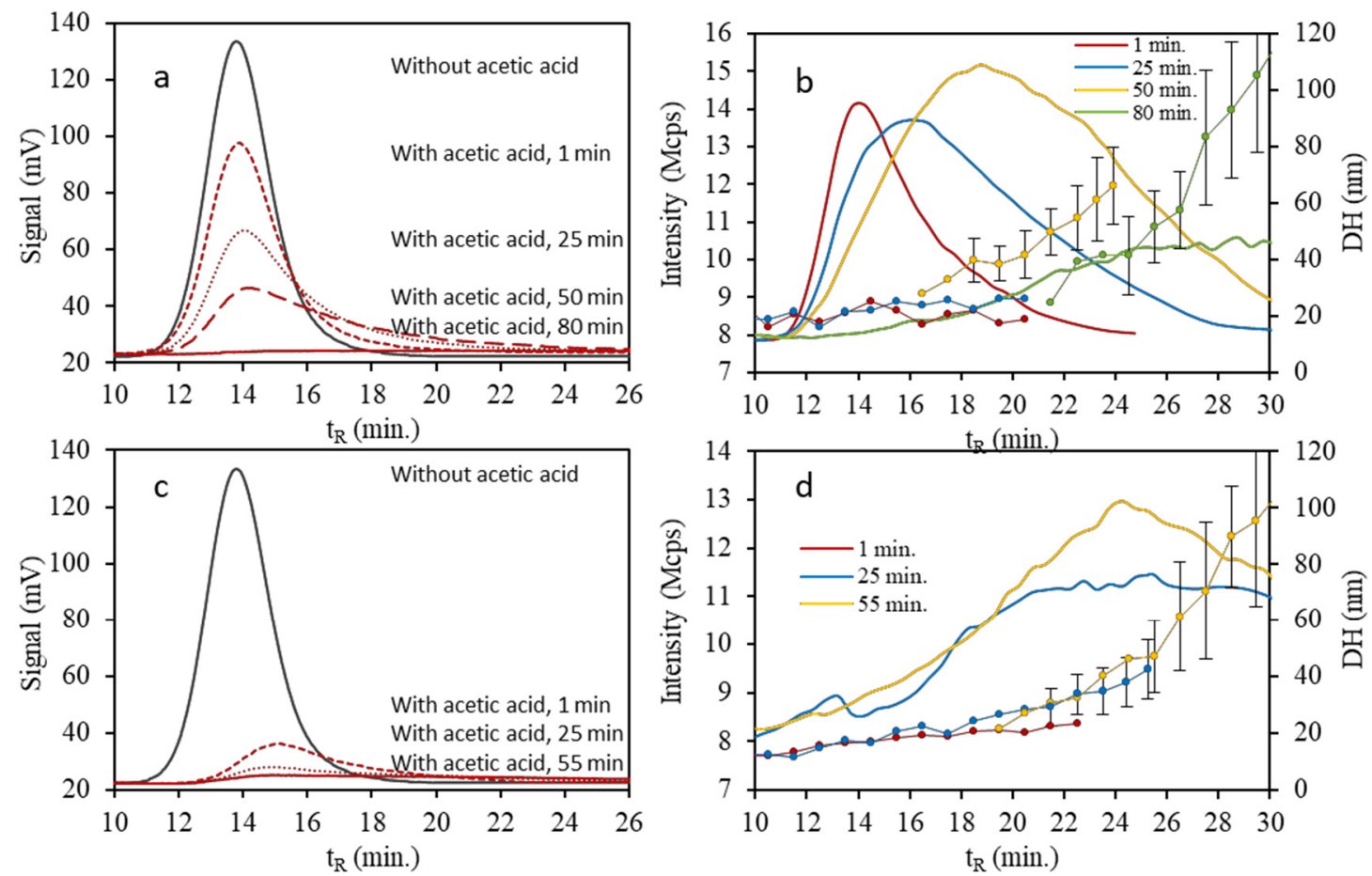

Figure 10. Fractograms wth UV-Vis detection corresponding to suspensions of AgNPs in a 1: 4 dilution $(5 \mu \mathrm{g} / \mathrm{mL})$ and $1.5 \mathrm{mM}(\mathbf{a})$ or $3 \mathrm{mM}(\mathbf{c})$ acetic acid at different times. Fractograms with DLS detection and hydrodynamic diameter $(\mathrm{DH})$ (confidence interval $\alpha=0.05$ ) corresponding to the same suspensions at different times $(\mathbf{b}, \mathbf{d})$ respectively. 
Under acidic $\mathrm{pH}$ conditions, the citrate capping, which provides stability to AgNPs, progressively loses its effectiveness by decreasing the surface negative charge density; the zeta potential in the interface that separates the Stern layer and the diffuse layer is less negative by reducing the $\mathrm{pH}$ of the medium $[27,28]$. NPs lose the repulsive forces that counteract the attractive van der Waals forces by removing the surface coating that protects them, thus facilitating their aggregation. It is observed with the exposure time that fractograms with a lower intensity of light scattering are recorded by the DLS detector as it can be seen in Figure 10b, which occurs more rapidly at lower $\mathrm{pH}$ (see Figure 10d). A greater tendency to oxidation of the NPs and release to the environment in the form of $\mathrm{Ag}^{+}$as the $\mathrm{pH}$ decreases is also described [31]. However, it must also be taken into account that the aggregation process implies the formation of sets of NPs from others of a smaller size, which can lead to a significant reduction in their total number. Specifically, for the formation of $100 \mathrm{~nm}$ NPs dozens of $20 \mathrm{~nm}$ NPs may be necessary depending on the morphology of the final aggregates. This fact can also cause a decrease in the intensity of scattered light registered by the DLS detector when aggregates of size much larger than the initial NPs are formed. Thus, these aggregates can cause a greater scattering of light, but there are much fewer of them.

In this sense, the response of the AgNPs to the action of different acids, consists of the loss of protection against the aggregation process, which can be observed by the decrease and the bathochromic shift in the plasmon (SPB) using UV-Vis spectroscopy, related with the increase in retention times, loss of area and higher hydrodynamic diameter values showed by AF4-UV-Vis-DLS. Likewise, reducing the $\mathrm{pH}$ of the suspensions increased the aggregation speed, as well as it can facilitate the oxidation and /or dissolution of the AgNPs surface. These statements should be considered for developing plasmonic assays for quantifying analytes properly.

Figure 11 shows the normalized absorbance values obtained by UV-Vis spectrometry at different times for each of assayed suspension against the concentration of acetic acid (11a) and hydrochloric acid (11d). A sinusoidal response is observed which for a certain range of concentrations, offers linearity in the change trend of absorbance with the acid concentration at a given time. This trend constitutes the basis on which plasmonic assays for the detection and/or quantification of substances are based [32,33]. Other possibilities are transforming the concentrations in their logs (see Figure 11b) or employing the maximum wavelength of the aggregated NPs for quantifying as it is done for acetic acid calibration (see Figure 11c). For all types of calibration graphs, the time influenced their equation and also the linear interval of concentrations (see Figure 11b,e), sensitivity and accuracy of the assay as can be reflected in Figure 11. Those aspects should be considered when the plasmonic assay is carried out.

Mainly, the application of AF4-UV-Vis-DLS provides new information about transformations that analytes induce in NPs and aid to fix the experimental variables, which must be controlled for obtaining reliable results. Besides, taking into account the great discrimination capacity of AF4 for explaining transformations generated in aqueous dispersions of NPs, their use could increase assay sensitivity if necessary. Please note that the dispersions assayed by AF4 corresponded to the lower concentrations tested by UV-Vis spectroscopy (see Figures 10 and 11 for comparison). In this sense, stablishing the equation of the AF4 calibration graphs for plasmonic assays is outside the goal of this paper. 

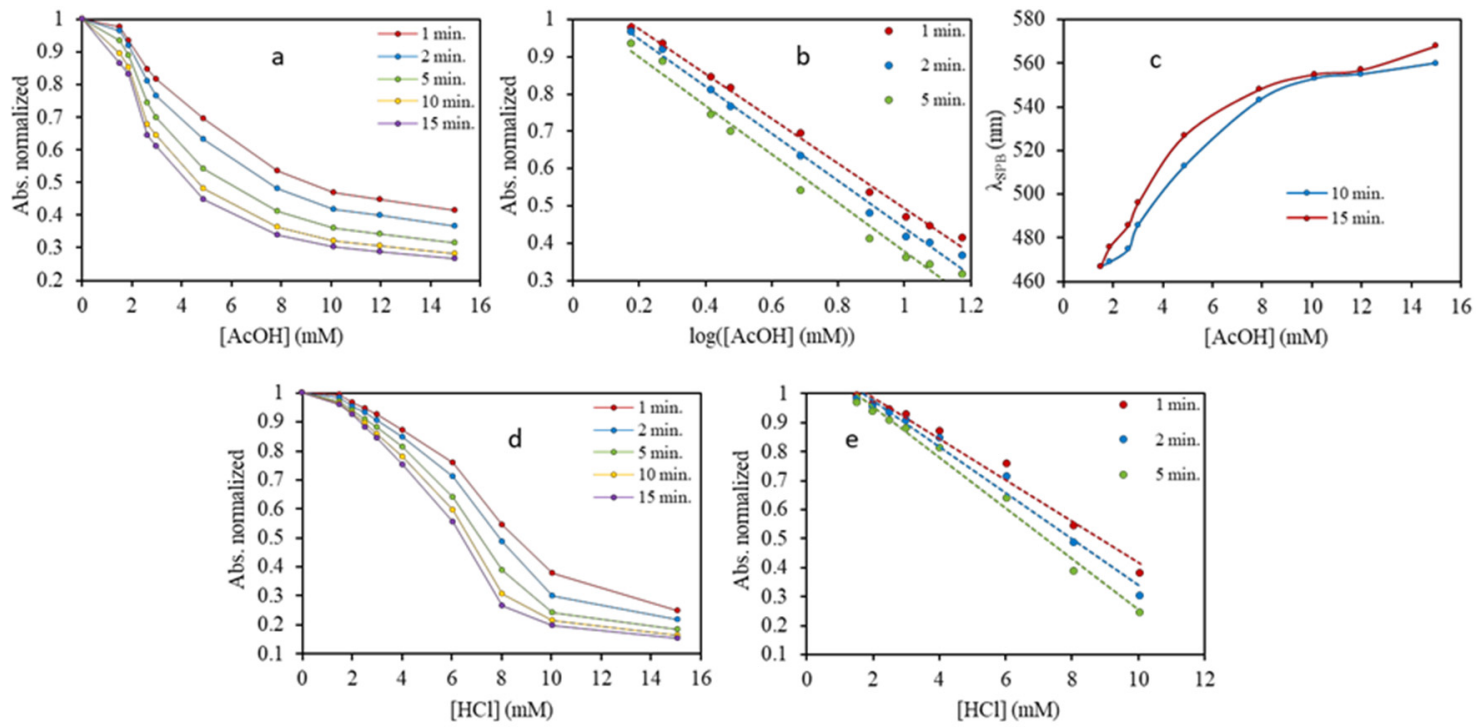

Figure 11. Normalized absorbance values at various times with the concentration of acetic acid (a), its $\log (\mathbf{b})$ of AgNPs diluted 1:4 $(5 \mu \mathrm{g} / \mathrm{mL})$ and evolution of the wavelength of the second plasmon maximum in the UV-Vis spectra at 10 and $15 \mathrm{~min}$ of its preparation $(\mathbf{c})$. (d,e) correspond to the influence of hydrochloric acid.

\section{Conclusions}

In this work, AF4 on-line coupled with UV-Vis and DLS detector was used to evaluate the impact of aqueous dilution on analytical responses of AuNPs and AgNPs dispersions. Different size dispersions were analyzed and the evolution of the fractograms profiles have been studied. The results demonstrated that for citrate-capped AuNPs and AgNPs, there was a decrease in the absorbance signal with time that depended on the particle size, this decrease was higher for bigger NPs. By another hand, hydrodynamic diameter was constant over dilution. AF4-UV-Vis-DLS showed that there is an effective variation in the number of NPs dispersed as a function of time, this variation was attributed to their dissolution in aqueous diluted dispersions. Comparing citrate-capped AgNPs and AuNPs, dissolution of the former NPs is more favored due to their lower stability, and the decrease on the absorbance signal as a function of time was more drastic. Hence, it can be concluded that AuNPs and AgNPs stability varied in diluted dispersions, and therefore their stability must be guarantee in order to obtain reliable results. In this context, AF4-UV-Vis-DLS demonstrated to be a potential tool to monitor variations in aqueous dispersions of metallic NPs, useful for understanding plasmonic assays and carrying out them properly. Other aspect to consider is the stability of the batches, it is demonstrated that the interaction of the batch with atmosphere modifies the number of NPs.

Supplementary Materials: The following are available online at http:/www.mdpi.com/2079-4991/10/9/1802/ s1, S.1.: Table S1. Method parameters for AuNPs. Table S2. AF4 method parameters for AgNPs. S.2.: Table S3. Hydrodynamic diameters for AuNPs dispersions as a function of dilution preparation time. Table S4. Hydrodynamic diameters for AgNPs dispersions as a function of dilution preparation. Figure S1. DLS spectra for AuNPs dispersions as a function of dilution preparation time. Figure S2. DLS spectra for AgNPs dispersions as a function of dilution preparation time. S.3.: Figure S3. Study of AuNPs-PBS dispersions as function of dilution preparation time.

Author Contributions: Conceptualization, P.C.-F.; Investigation, P.C.-F., L.S.-N., A.B.-P. and Y.M.-M.; Methodology, L.S.-N., A.B.-P. and Y.M.-M.; Resources, P.C.-F.; Supervision, P.C.-F. All authors have read and agreed to the published version of the manuscript.

Funding: EU FEDER and the Gobierno de España MCIU-AEI (CTQ2017-90082-P), the Generalitat Valenciana (PROMETEO Program 2020/078) and EU-FEDER-Generalitat Valenciana (IDIFEDER/2018/049). L. Sanjuan-Navarro expresses his gratitude for the FPU-grant 17-01655 (MCIU-AEI).

Conflicts of Interest: The authors declare no conflict of interest. 


\section{References}

1. Hinterwirth, H.; Wiedmer, S.K.; Moilanen, M.; Lehner, A.; Allmaier, G.; Waitz, T.; Linder, W.; Lämmerhofer, M. Comparative method evaluation for size and size-distribution analysis of gold nanoparticles. J. Sep. Sci. 2013, 36, 2952-2961. [CrossRef]

2. Hone, D.C.; Walker, P.I.; Evans-Gowing, R.; FitzGerlad, S.; Beeby, A.; Chambrier, I. Generation of cytotoxic singlet oxygen via phthalocyanine-stabilized gold nanoparticles: A potential delivery vehicle for photodynamic therapy. Langmuir 2002, 18, 2985-2987. [CrossRef]

3. Hutter, E.; Maysinger, D. Gold nanoparticles and quantum dots for bioimaging. Microsc. Res. Techniq. 2011, 74, 592-604. [CrossRef]

4. Kneipp, J.; Kneipp, H.; Rice, W.L.; Kneipp, K. Optical probes for biological applications based on surface-enhanced raman scattering from indocyanine green on gold nanoparticles. Anal. Chem. 2005, 77, 2381-2385. [CrossRef]

5. Saenmuangchin, R.; Siripinyanond, A. Flow field-flow fractionation for hydrodynamic diameter estimation of gold nanoparticles with various types of surface coatings. Anal. Bioanal. Chem. 2018, 410, 6845-6859. [CrossRef]

6. Gour, A.; Jain, N.K. Advances in green synthesis of nanoparticles. Artif. Cells Nanomed. Biotechnol. 2019, 47, 844-851. [CrossRef]

7. Chugh, H.; Sood, D.; Chandra, I.; Tomar, V.; Dhawan, G.; Chandra, R. Role of gold and silver nanoparticles in cancer nano-medicine. Artif. Cells Nanomed. Biotechnol. 2018, 46, 1210-1220. [CrossRef]

8. González-Fuenzalida, R.A.; Sanjuan-Navarro, L.; Moliner-Martínez, Y.; Campíns-Falcó, P. Quantitative study of the capture of silver nanoparticles by several kinds of soils. Sci. Total Environ. 2018, 630, 1226-1236. [CrossRef]

9. Dueñas-Mas, M.J.; Soriano, M.L.; Ruiz-Palomero, C.; Valcárcel, M. Modified nanocellulose as promising material for the extraction of gold nanoparticles. Microchem. J. 2018, 138, 379-383. [CrossRef]

10. González-Fuenzalida, R.A.; Moliner-Martínez, Y.; Molins-Legua, C.; Campíns-Falcó, P. Miniaturized liquid chromatography coupled on-line to in-tube solid-pase microextraction for characterization of metallic nanoparticles using plasmonic measurements. A tutorial. Anal. Chim. Acta 2019, 1045, 23-41. [CrossRef]

11. Meisterjahn, B.; Wagner, S.; Kammer, F.; Hennecke, D.; Hofmann, T. Silver and gold nanoparticles separation using asymmetrical flow-field flow fractionation: Influence of run conditions and of particle membrane charges. J. Chromatogr. A 2016, 1440, 150-159. [CrossRef]

12. Malik, M.I.; Pasch, H. Field-flow fractionation: New and exciting perspectives in polymer analysis. Prog. Polym. Sci. 2016, 63, 42-85. [CrossRef]

13. Irfan, M.; Moniruzzaman, M.; Ahmad, T.; Osman, O.Y.; Mandal, P.C.; Bhattacharjee, S.; Hussain, M. Stability, interparticle interactions and catalytic performance of gold nanoparticles synthesized through ionic liquid mediated oil palm leaves extract. J. Environ. Chem. Eng. 2018, 6, 5024-5031. [CrossRef]

14. Rouhaba, L.L.; Jaber, J.A.; Schlenoff, J.B. Aggregation-Resistant Water-Soluble Gold Nanoparticles. Langmuir 2007, 23, 12799-12801.

15. Baalousha, M.; Arkill, K.P.; Romer, I.; Palmer, R.E.; Lead, J.R. Transformations of citrate and Tween coated silver nanoparticles reacted with $\mathrm{Na}_{2}$ S. Sci. Total Environ. 2015, 502, 344-353. [CrossRef]

16. González-Fuenzalida, R.A.; Moliner-Martínez, Y.; González-Béjar, M.; Molins-Legua, C.; Verdú-Andrés, J.; Pérez-Prieto, J.; Campíns-Falcó, P. In Situ Colorimetric Quantification of Silver Cations in the Presence of Silver Nanoparticles. Anal. Chem. 2013, 85, 10013-10016. [CrossRef]

17. Calzolai, L.; Gilliland, D.; Pascual Garcia, C.; Rossi, F. Separation and characterization of gold nanoparticle mixtures by flow-field-flow fractionation. J. Chromatogr. A 2011, 1218, 4234-4239. [CrossRef]

18. Jochem, A.R.; Ankah, G.N.; Meyer, L.A.; Elsenberg, S.; Johann, C.; Kraus, T. Colloidal Mechanisms of Gold Nanoparticles Loss in Asymmetric Flow Field-Flow Fractionation. Anal. Chem. 2016, 88, 10065-10073. [CrossRef]

19. Chang, Y.J.; Shih, Y.H.; Su, C.H.; Ho, H.C. Comparison of three analytical methods to measure the size of silver nanoparticles in real environmental water and wastewater samples. J. Hazard. Mater. 2017, 322, 95-104. [CrossRef] 
20. Yang, Y.; Long, C.L.; Li, H.P.; Wang, Q.; Yang, Z.G. Analysis of silver and gold nanoparticles in environmental water using single particle-inductively coupled plasma-mass spectrometry. Sci. Total Environ. 2016, 563, 996-1007. [CrossRef]

21. Baiee, R.; Liu, Z.; Li, L. Understanding the stability and durability of laser-generated Ag nanoparticles and effects on their antibacterial activities. Adv. Nat. Sci Nanosci. 2019, 10, 035001. [CrossRef]

22. Espinoza, M.G.; Hinks, M.L.; Mendoza, A.M.; Pullman, D.P.; Peterson, K.I. Kinetics of Halide-Induced Decomposition and Aggregation of Silver Nanoparticles. J. Phys. Chem. C 2012, 116, 8305-8313. [CrossRef]

23. Li, X.; Lenhart, J.J.; Walker, H.W. Dissolution-Accompanied Aggregation Kinetics of Silver Nanoparticles. Langmuir 2010, 26, 16690-16698. [CrossRef] [PubMed]

24. Henglein, A. Colloidal Silver Nanoparticles: Photochemical Preparation and Interaction with O2, CCl4, and Some Metal Ions. Chem. Mater. 1998, 10, 444-450. [CrossRef]

25. Yin, Y.; Li, Z.; Zhong, Z.; Gates, B.; Xia, Y.; Venkateswaran, S. Synthesis and characterization of stable aqueous dispersions of silver nanoparticles through the Tollens process. J. Mater. Chem. 2002, 12, 522-527. [CrossRef]

26. Axson, J.L.; Stark, D.I.; Bondy, A.L.; Capracotta, S.S.; Maynard, A.D.; Philbert, M.A.; Bergin, I.L.; Ault, A.P. Rapid Kinetics of Size and pH-Dependent Dissolution and Aggregation of Silver Nanoparticles in Simulated Gastric Fluid. J. Phys. Chem. C 2015, 119, 20632-20641. [CrossRef]

27. Prathna, T.C.; Chandrasekaran, N.; Mukherjee, A. Studies on aggregation behaviour of silver nanoparticles in aqueous matrices: Effect of surface functionalization and matrix composition. Colloids Surf. A Physicochem. Eng. Asp. 2011, 390, 216-224. [CrossRef]

28. El-Badawy, A.M.; Luxton, T.P.; Silva, R.G.; Scheckel, K.G.; Suidan, M.T.; Tolaymat, T.M. Impact of Environmental Conditions ( $\mathrm{pH}$, Ionic Strength, and Electrolyte Type) on the Surface Charge and Aggregation of Silver Nanoparticles Suspensions. Environ. Sci. Technol. 2010, 44, 1260-1266. [CrossRef]

29. Rogers, K.R.; Bradham, K.; Tolaymat, T.; Thomas, D.J.; Hartmann, T.; Ma, L.; Williams, A. Alterations in physical state of silver nanoparticles exposed to synthetic human stomach fluid. Sci. Total Environ. 2012, 420, 334-339. [CrossRef]

30. Mwilu, S.K.; El-Badawy, A.M.; Bradham, K.; Nelson, C.; Thomas, D.; Scheckel, K.G.; Tolaymat, T.; Ma, L.; Rogers, K.R. Changes in silver nanoparticles exposed to human synthetic stomach fluid: Effects of particle size and surface chemistry. Sci. Total Environ. 2013, 447, 90-98. [CrossRef]

31. Liu, J.; Hurt, R.H. Ion Release Kinetics and Particle Persistence in Aqueous Nano-Silver Colloids. Environ. Sci. Technol. 2010, 44, 2169-2175. [CrossRef] [PubMed]

32. Jornet-Martínez, N.; González-Béjar, M.; Moliner-Martínez, Y.; Campins-Falcó, P.; Pérez-Prieto, J. Sensitive and Selective Plasmonic Assay for Spermine as Biomarker in Human Urine. Anal. Chem. 2014, 86, 1347-1351. [CrossRef] [PubMed]

33. Jornet-Martínez, N.; Hakobyan, L.; Argente-García, A.I.; Molins-Legua, C.; Campins-Falcó, P. Nylon-Supported Plasmonic Assay Based on the Aggregation of Silver Nanoparticles: In Situ Determination of Hydrogen Sulfide-like Compounds in Breath Samples as a Proof of Concept. ACS Sens. 2019, 4, 2164-2172. [CrossRef] [PubMed]

(C) 2020 by the authors. Licensee MDPI, Basel, Switzerland. This article is an open access article distributed under the terms and conditions of the Creative Commons Attribution (CC BY) license (http://creativecommons.org/licenses/by/4.0/). 